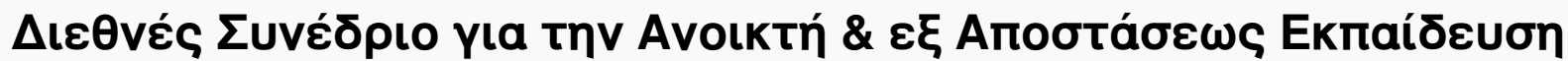

Tóp. 6, Ap. 2B (2011)

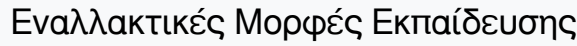

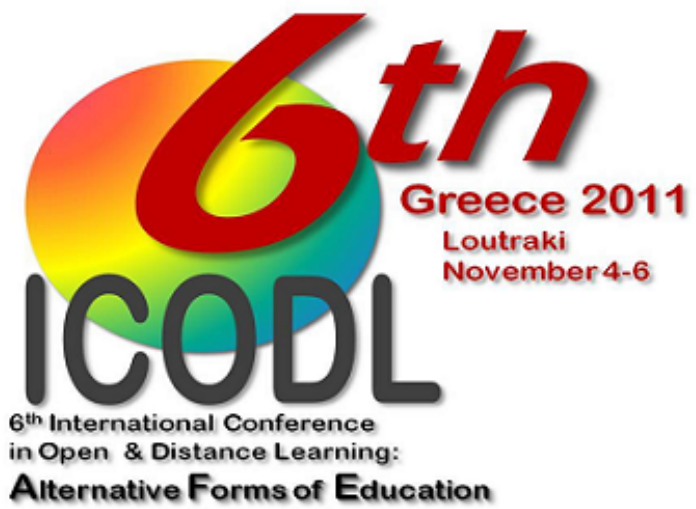

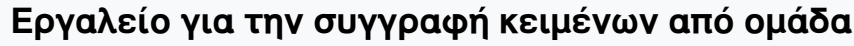

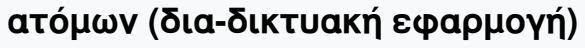

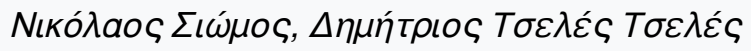

doi: $10.12681 /$ icodl. 670

TOMOE B

PART / MEPOE B 


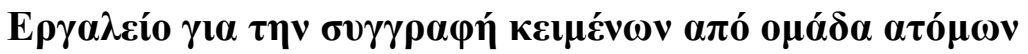

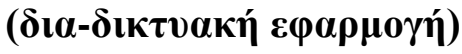

\section{Collaborative Writing tool (a web application)}

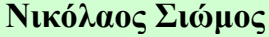 \\ siomosn@gmail.com
}

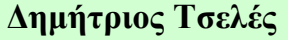

\begin{abstract}
The objective of this paper is to present the design of a web application, and results of its use, regarding collaborative writing. The objective of this web application is to help both students and professors to increase effectiveness, performance, save time, make their life easier yet it will provide a sophisticated way of their collaborative work. All users of the system will be able to share information and communicate over the internet in order to manage their ideas and mostly their writing process. Professors will be able to easily manage large amount of projects and save valuable time in order to achieve the best results. It was the late of 80 s when researchers found that a group's effort resulted in better document than when they worked alone and such collaborative writing tools be really helpful not only for educational staff but also for workers in operational departments. My personal experience in private sector as a team leader helped me to understand the importance of such tools and motivated me to start my research in this field. The purpose of this project is not to deprecate current technologies but to find better ways in collaborative writing.
\end{abstract}

\section{Пєрі́ $\eta \Psi \psi$}

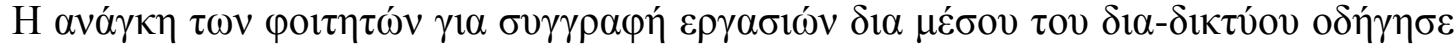

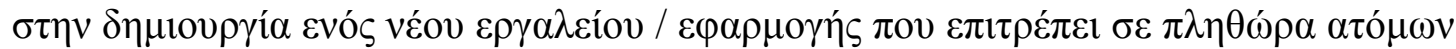

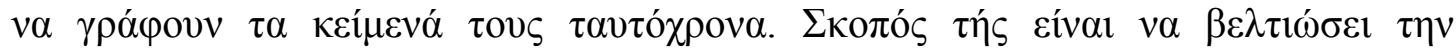

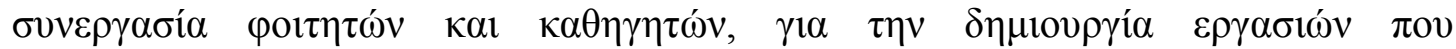

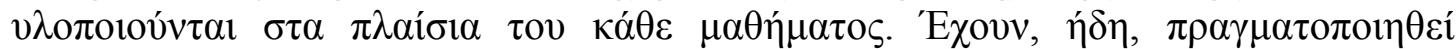

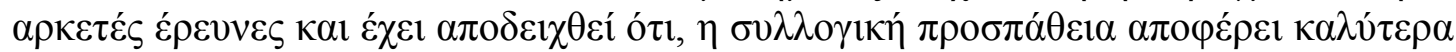

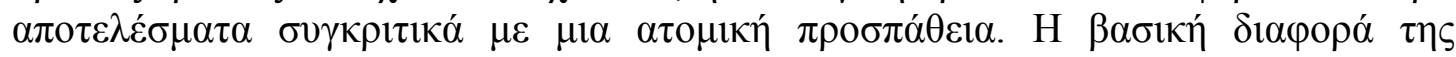

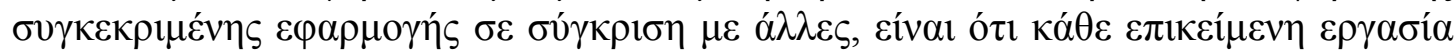

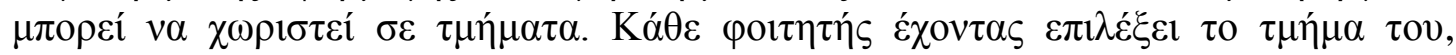

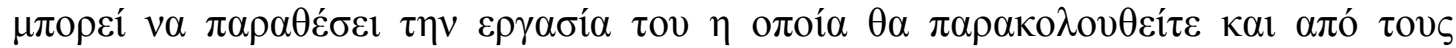

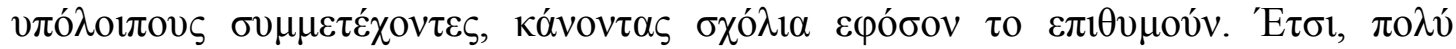

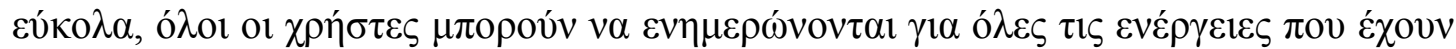

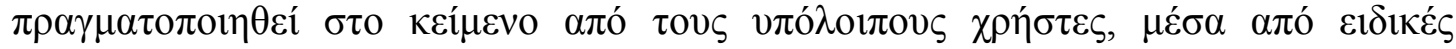

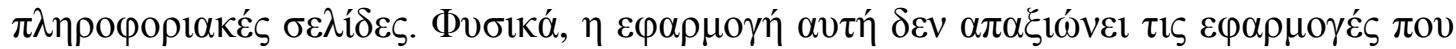

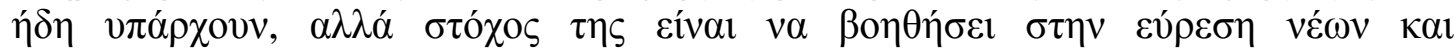

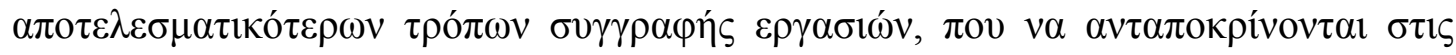
$\pi \rho \alpha \gamma \mu \alpha \tau 1 \kappa \varepsilon ́ \varsigma \alpha \nu \alpha ́ \gamma \kappa \varepsilon \varsigma \tau \omega \nu \chi \rho \eta \sigma \tau \omega ́ v$.

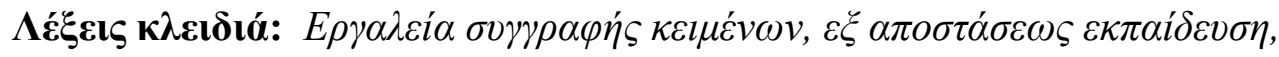

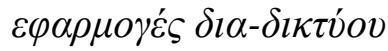




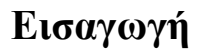

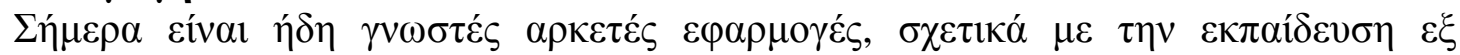

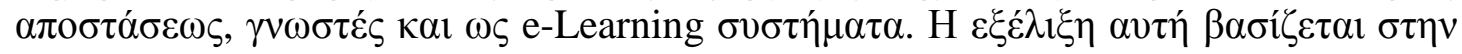

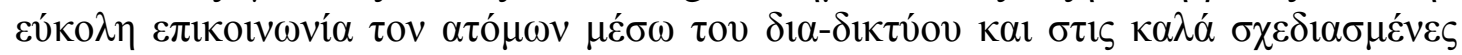

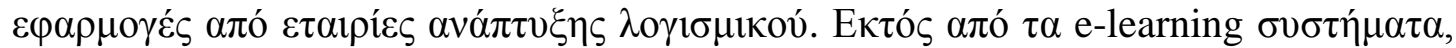

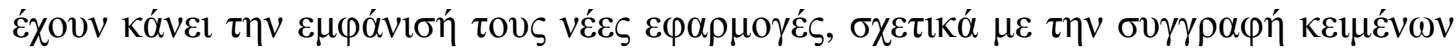

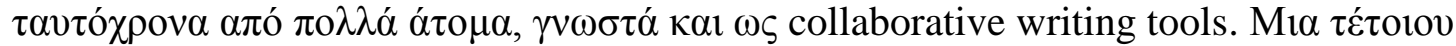

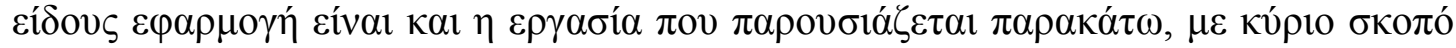

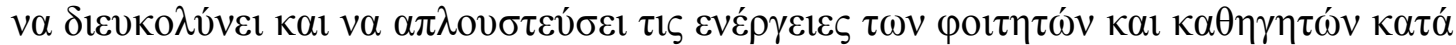

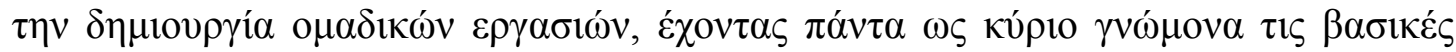
$\alpha \pi \alpha \iota \eta \dot{\sigma \varepsilon \varepsilon \varsigma \varsigma ~ \tau o v \varsigma . ~}$

\section{'Ep\&vva}

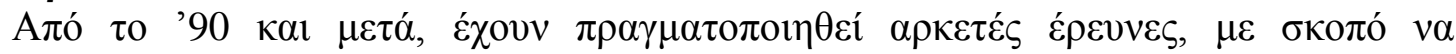

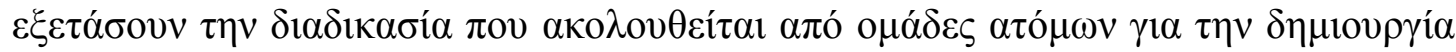

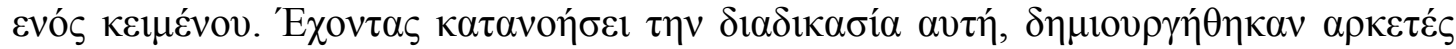

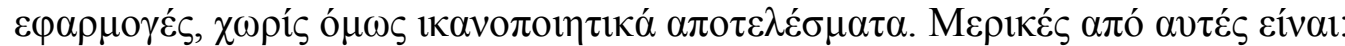

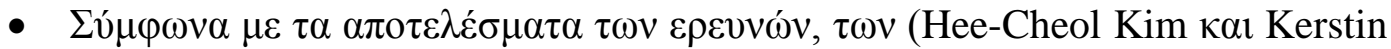

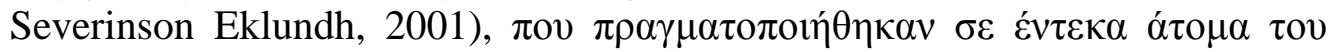

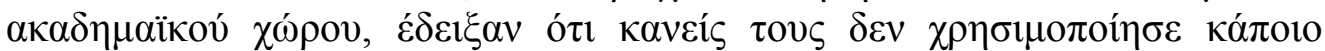

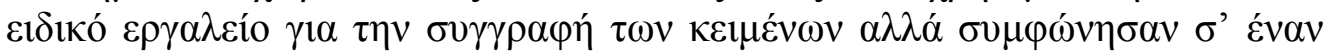

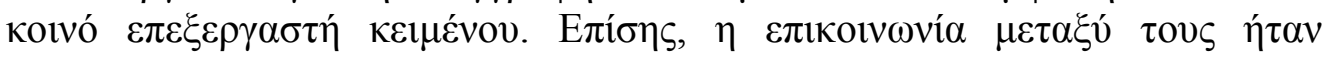

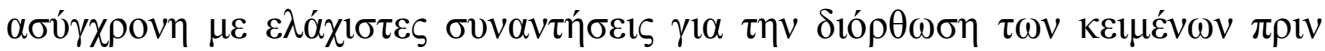
$\tau \eta \nu \pi \alpha \rho \alpha ́ \delta o \sigma \eta$.

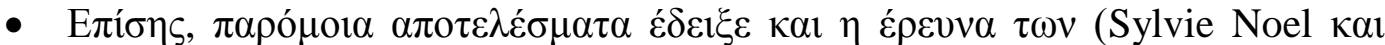

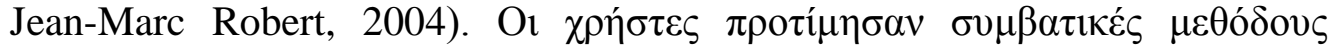

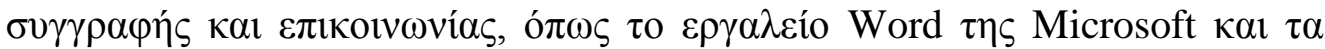

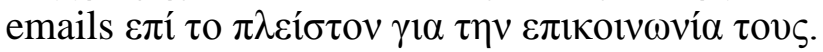

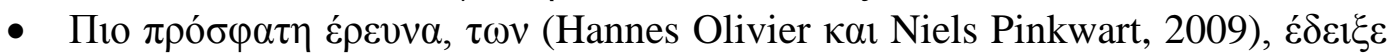

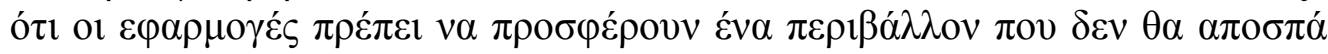

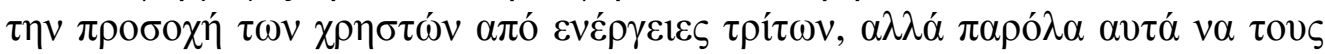

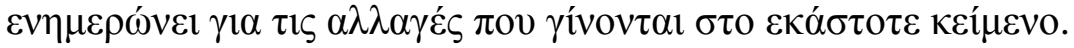

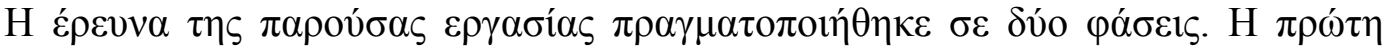

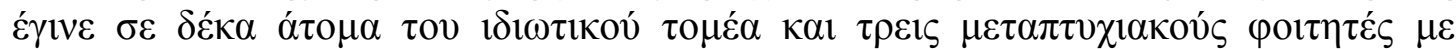

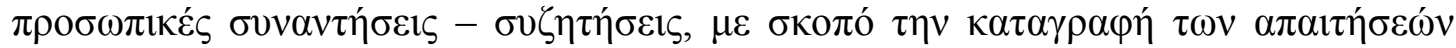

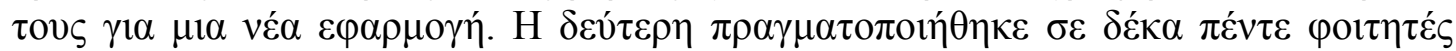

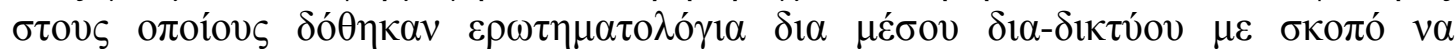

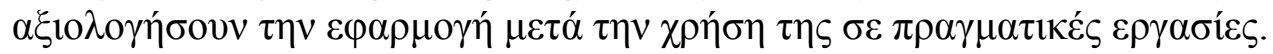

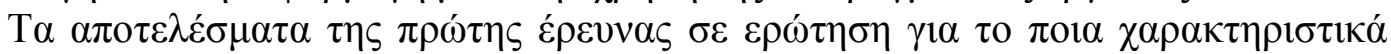

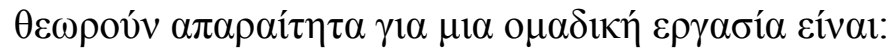

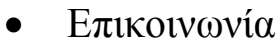

- $\Sigma \tau \rho \alpha \tau \eta \gamma 1 \kappa \eta ́ ~ \sigma u \gamma \gamma \rho \alpha \varphi \eta ́ s$

- $\Sigma \chi o ́ \lambda ı a$

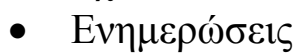

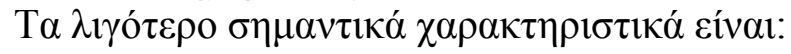

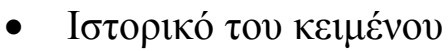

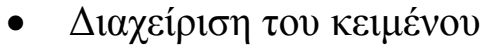

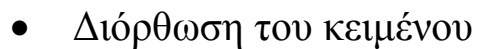




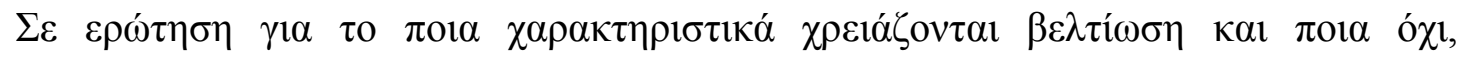
$\alpha \pi \alpha ́ v \tau \eta \sigma \alpha v:$

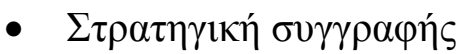

- $\Sigma \chi 0 ́ \lambda 1 \alpha$

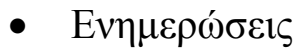

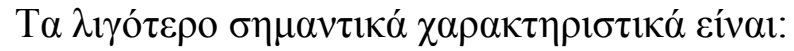

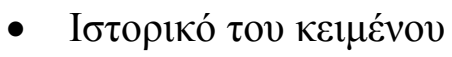

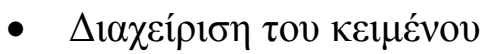

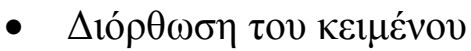

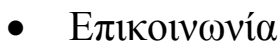

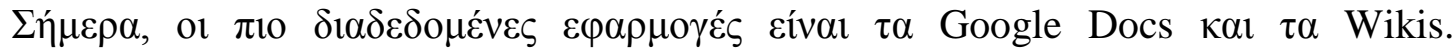

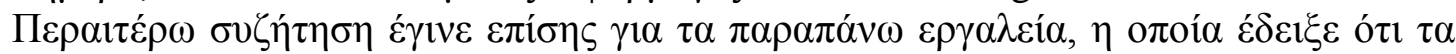

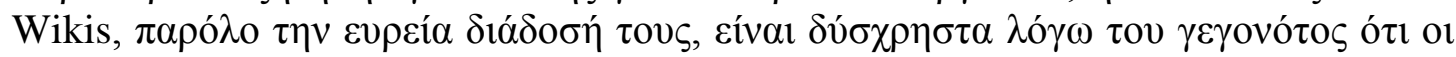

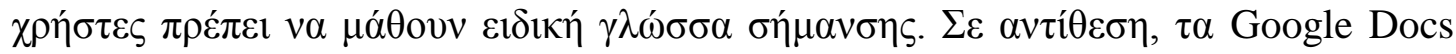

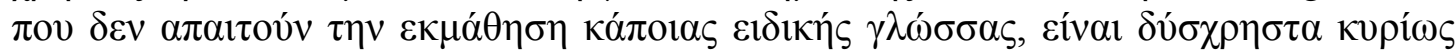

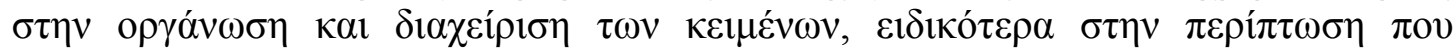

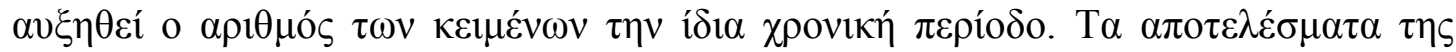

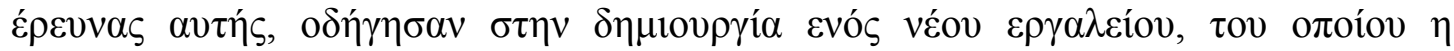

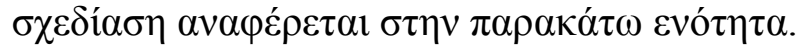

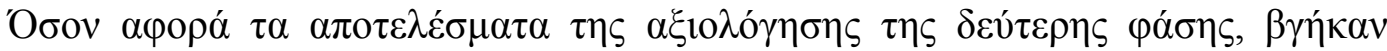

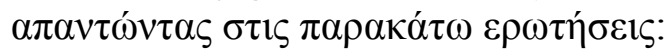

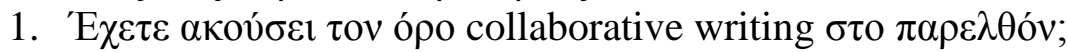

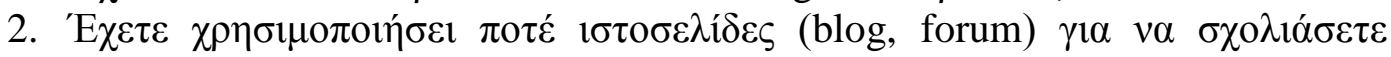

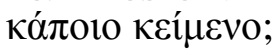

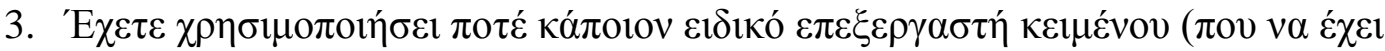

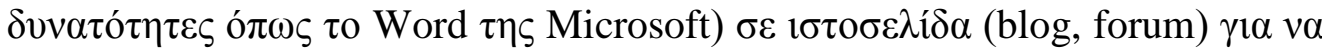

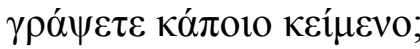

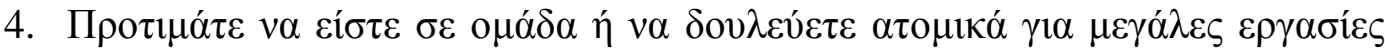

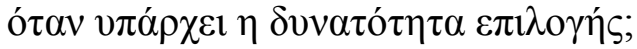

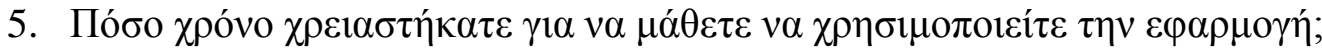

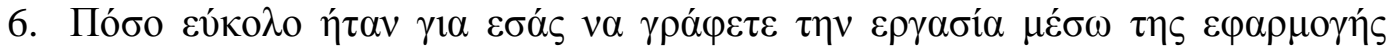

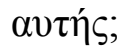

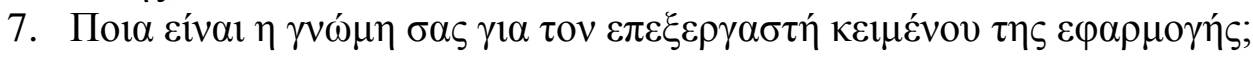

8. 'Н $\varepsilon \varphi \alpha \rho \mu о \gamma \eta ́ s ;$

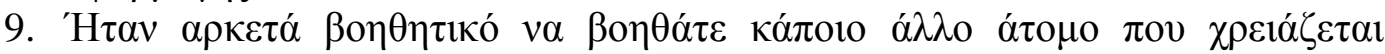

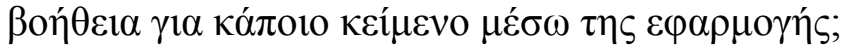

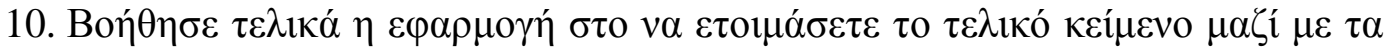

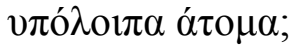

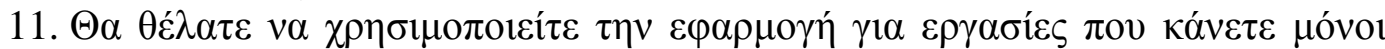
$\sigma \alpha \varsigma ;$

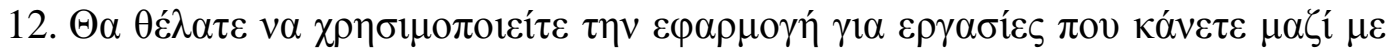

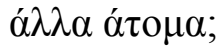

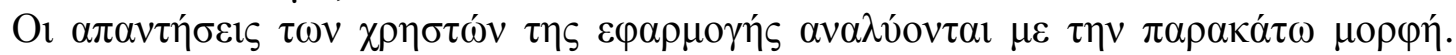
( 


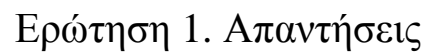

$\mathrm{A}=\mathrm{N} \alpha \mathbf{1}$

$\mathrm{B}={ }^{\prime} \mathrm{O} \chi 1$

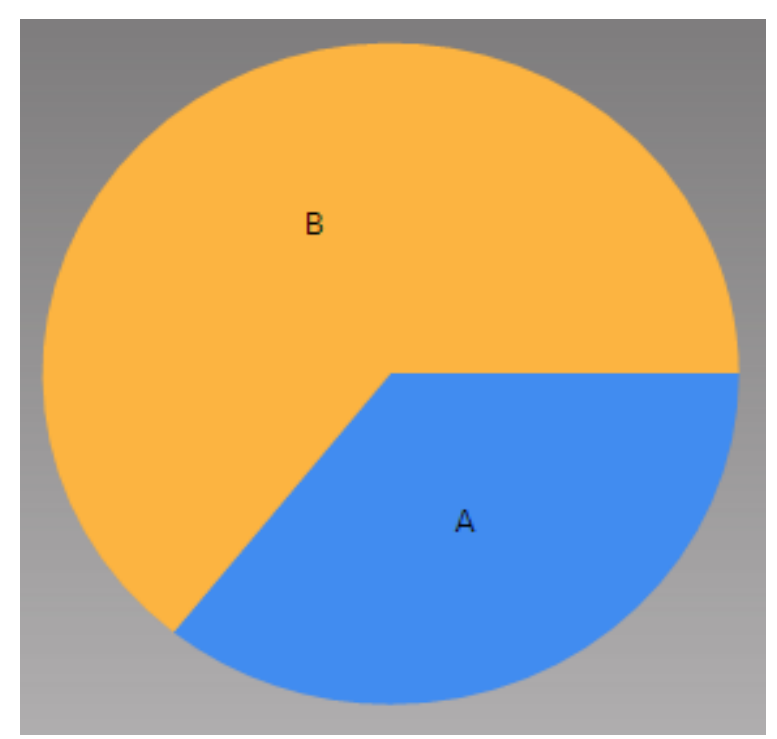

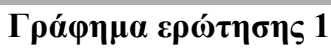

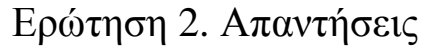

$\mathrm{A}=\mathrm{N} \alpha \mathrm{l}$

$\mathrm{B}={ }^{\prime} \mathrm{O} \chi 1$

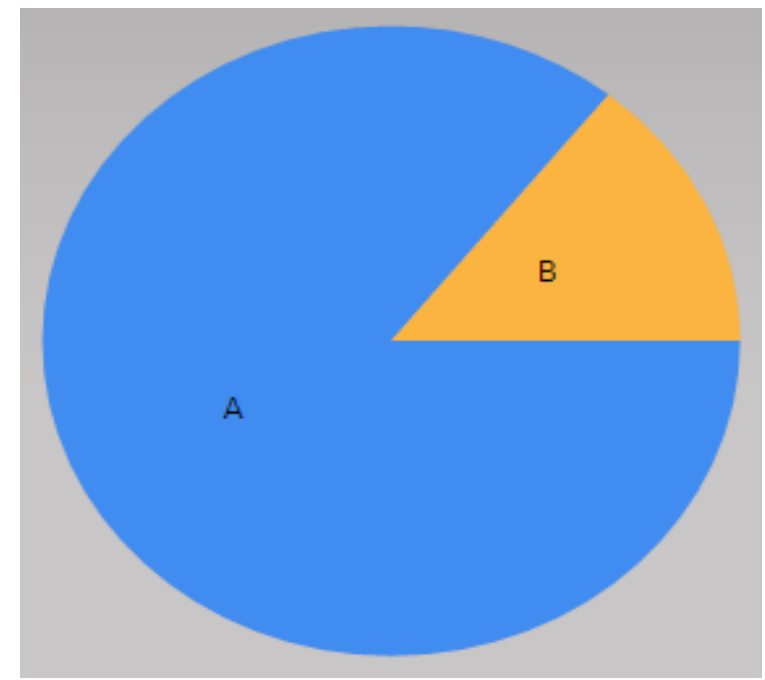

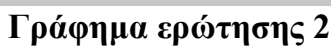

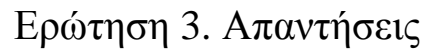

$\mathrm{A}=\mathrm{N \alpha}$

$\mathrm{B}={ }^{\prime} \mathrm{O} \chi 1$

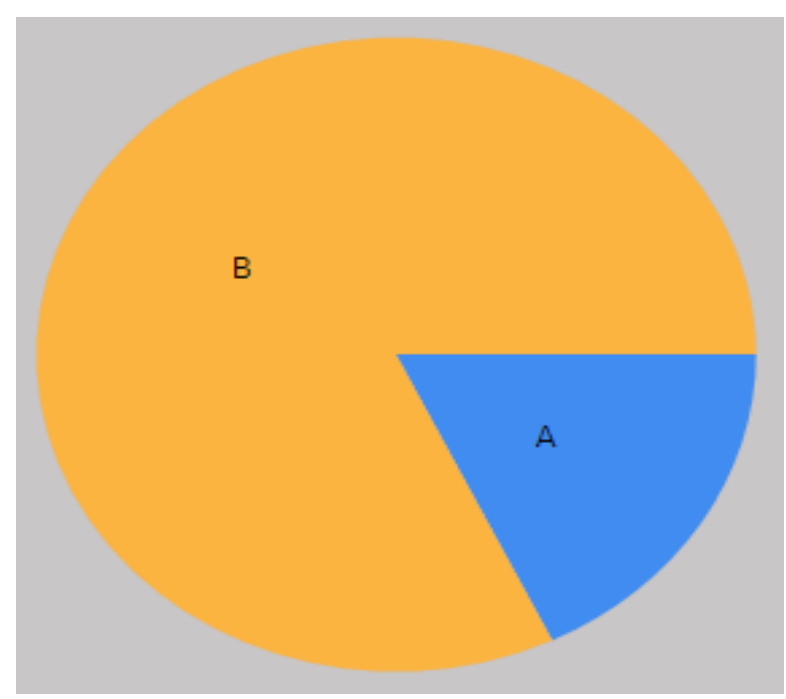

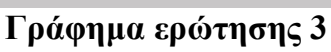

SECTION B: applications, experiences, good practices, descriptions and outlines, educational activities, issues for dialog and discussion 


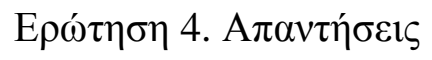

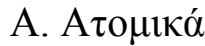
B. $\Sigma \varepsilon$ o $\mu \alpha \dot{\delta} \delta \alpha$

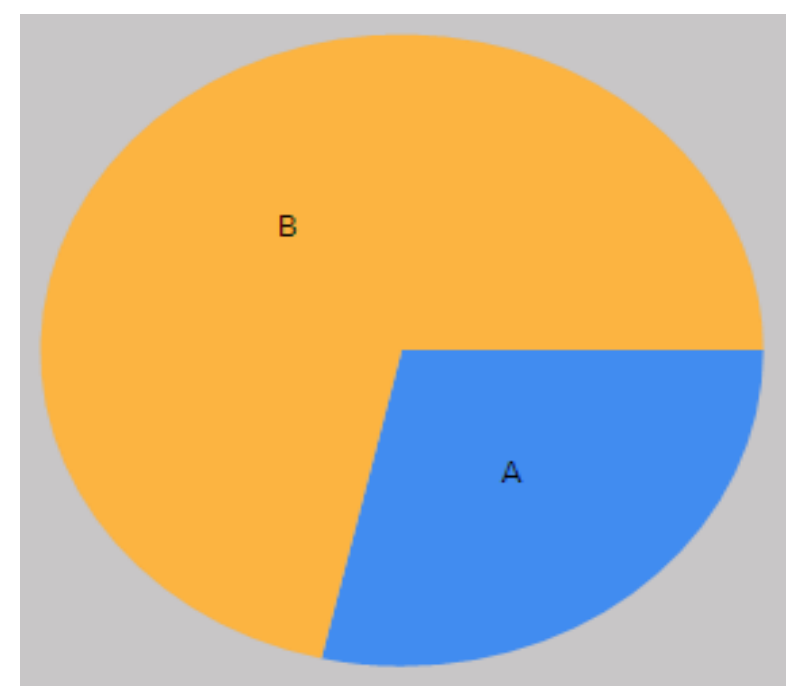

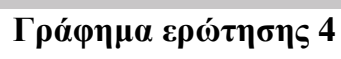

Е

$\mathrm{A}=5 \mu \varepsilon 10 \lambda \varepsilon \pi \tau \alpha \dot{\alpha}$

$\mathrm{B}=10 \mu \varepsilon 20 \lambda \varepsilon \pi \tau \dot{\alpha}$

$\Gamma=20 \mu \varepsilon 30 \lambda \varepsilon \pi \tau \alpha ́$

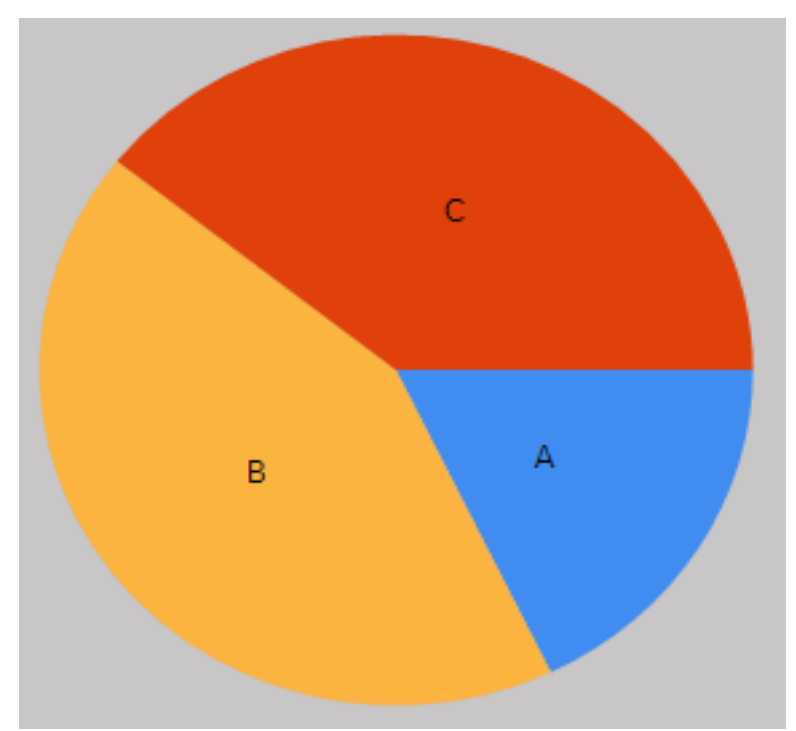

Гра́фๆ

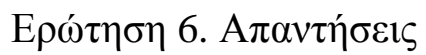

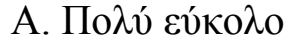
В. Еи́ко
Г. $\Delta v ́ \sigma \kappa о \lambda о$

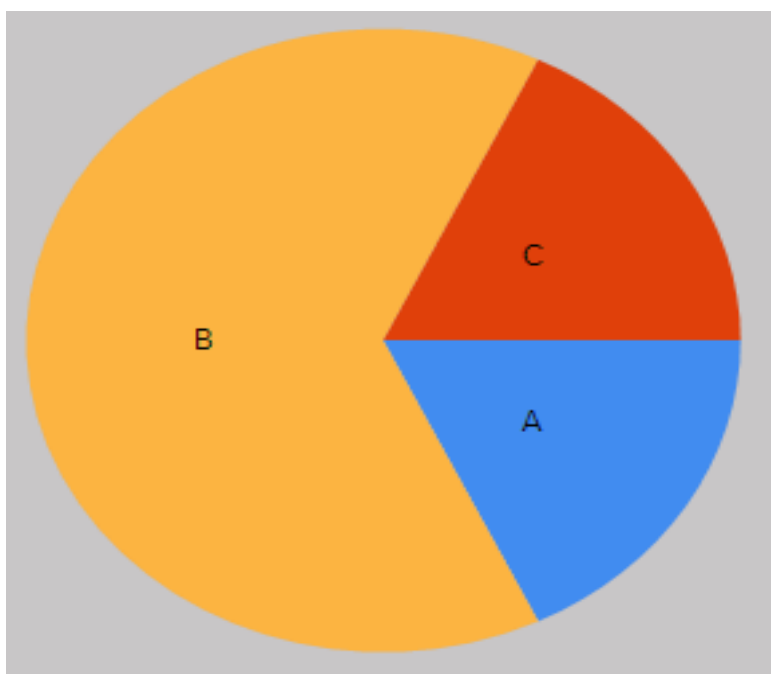

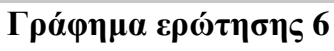




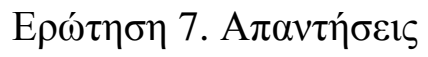

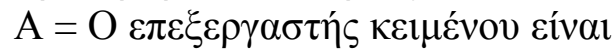

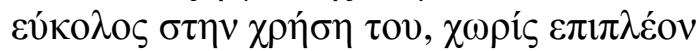
$\delta v v \alpha \tau o ́ \tau \eta \tau \varepsilon \varsigma$

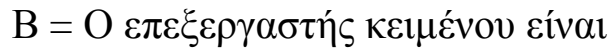

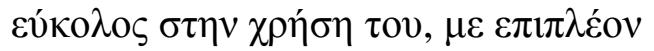

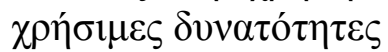

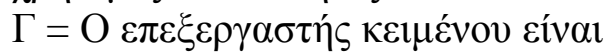

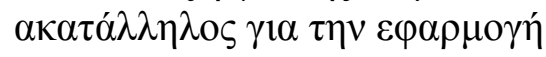

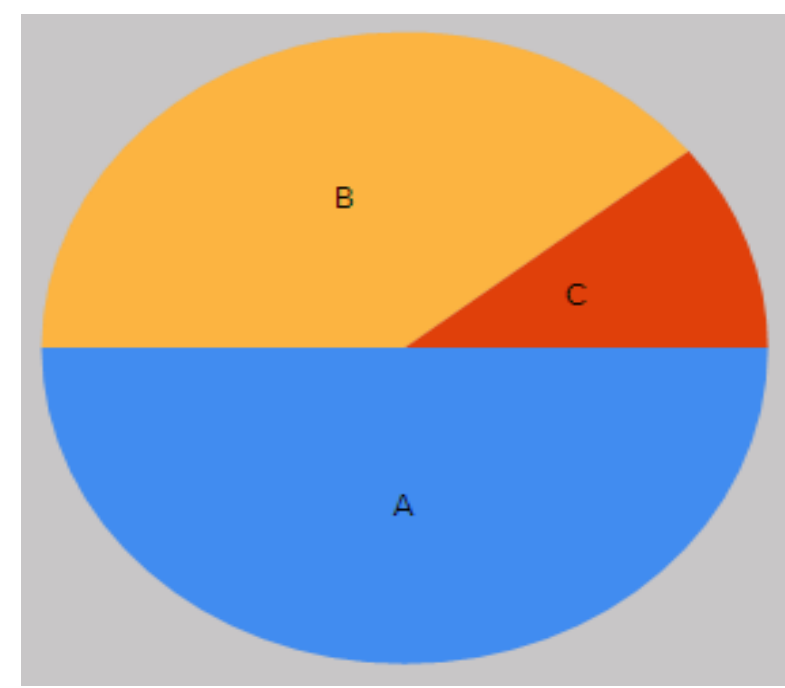

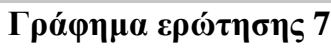

Е $\mathrm{A}=\mathrm{N \alpha}$ $\mathrm{B}=\mathrm{O} \chi \mathrm{l}$

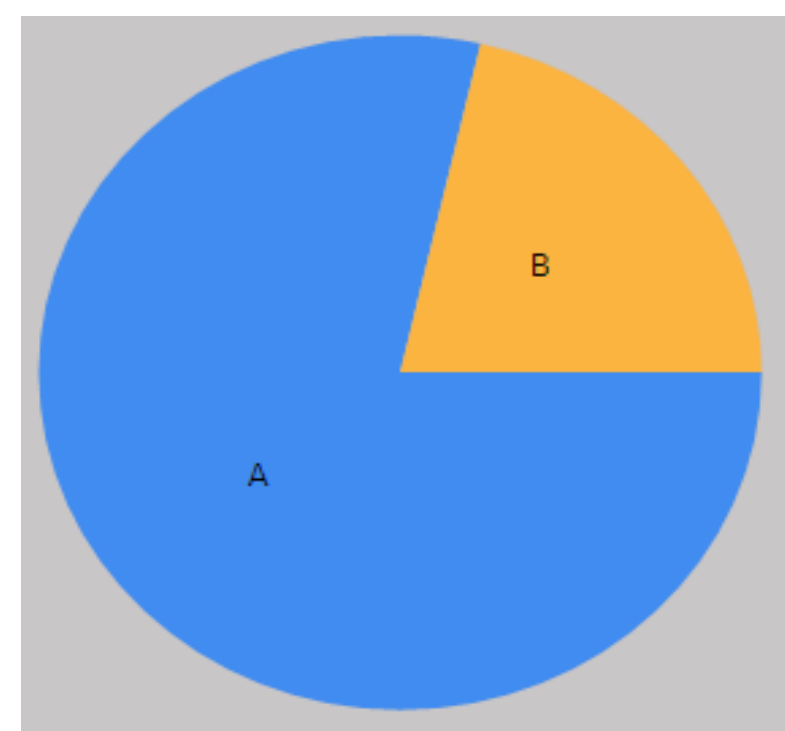

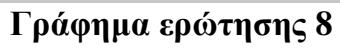

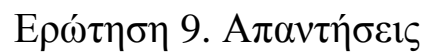

$\mathrm{A}=\mathrm{N} \alpha \mathrm{l}$

$\mathrm{B}=\mathrm{O}^{\mathrm{O}} \chi_{l}$

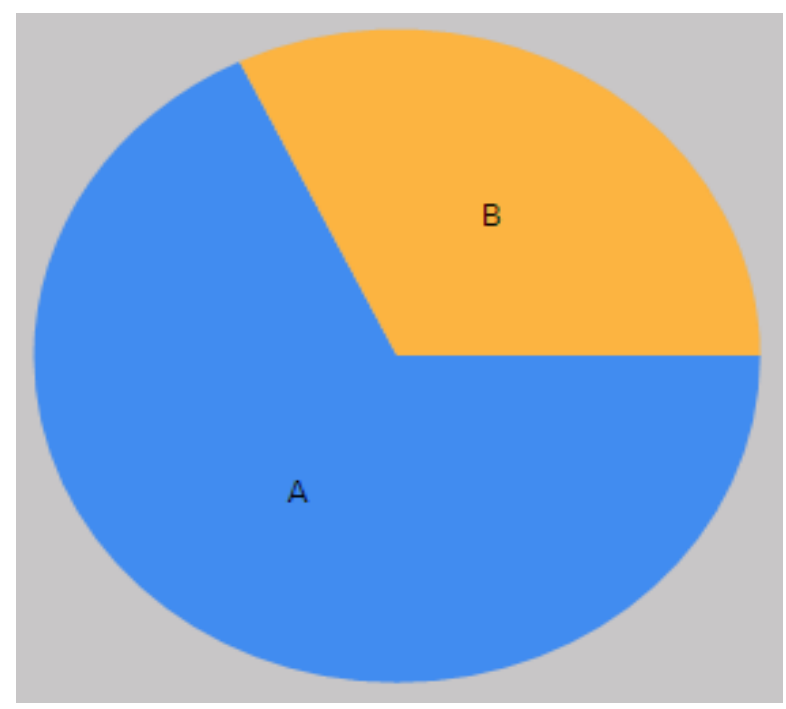

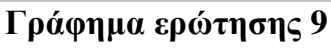




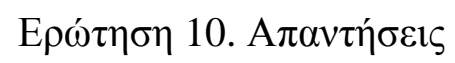

$\mathrm{A}=\mathrm{N \alpha l}$

$\mathrm{B}=\mathrm{O} \chi \mathrm{l}$

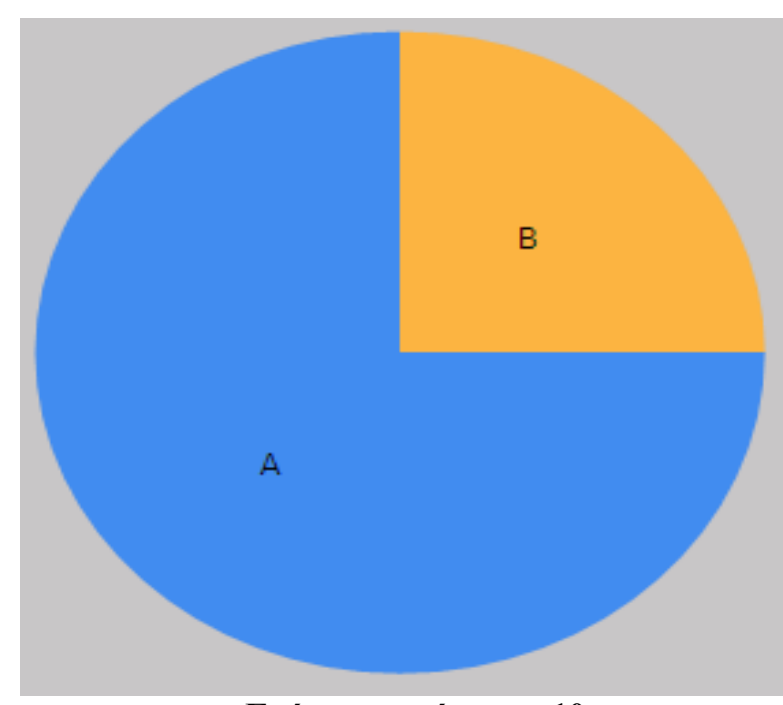

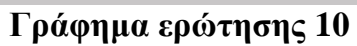

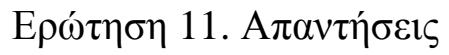

$\mathrm{A}=\mathrm{N \alpha l}$

$\mathrm{B}={ }^{\prime} \mathrm{O} \chi 1$

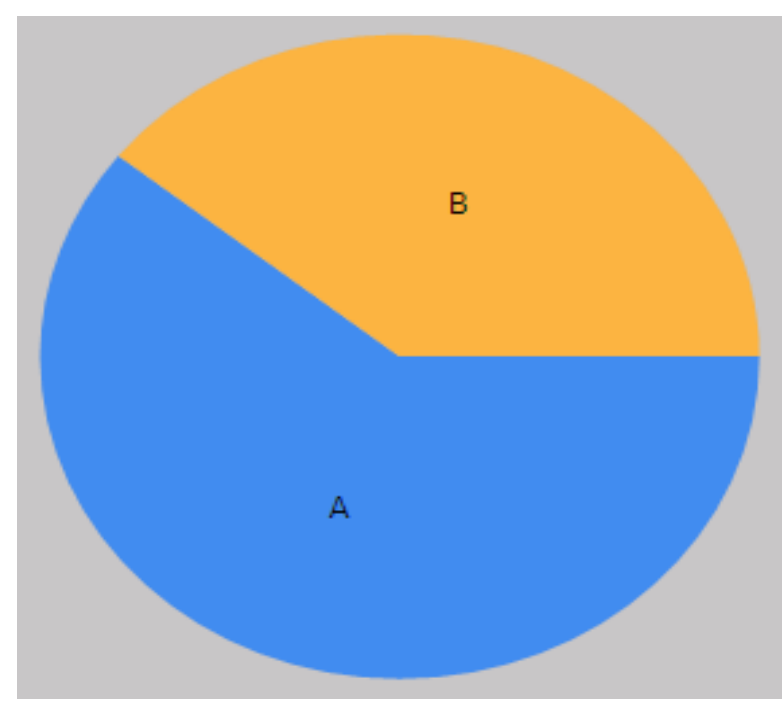

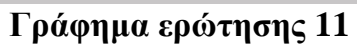

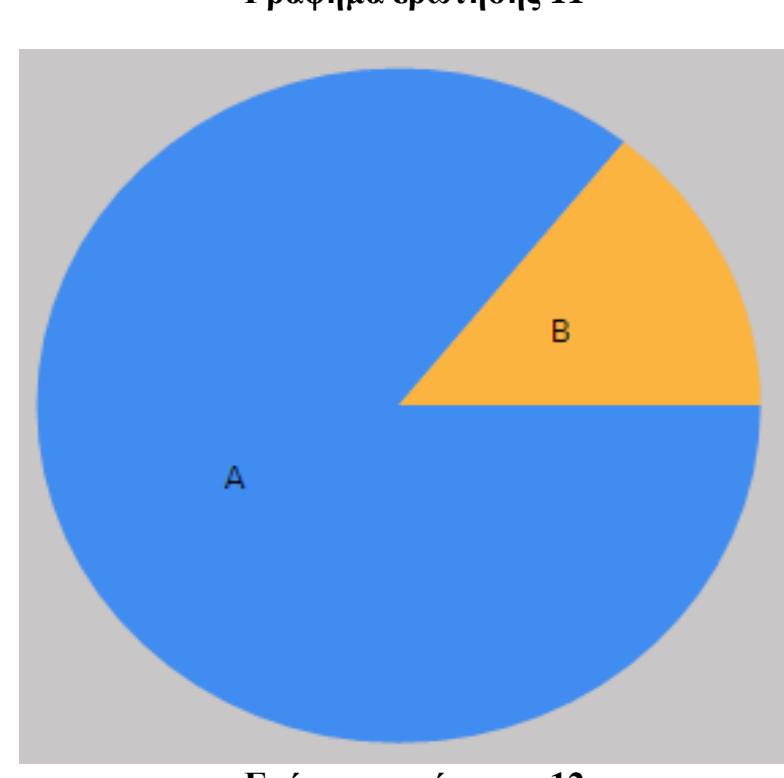

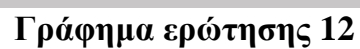

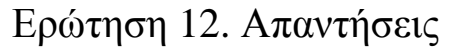

$\mathrm{A}=\mathrm{N} \alpha \iota$

$\mathrm{B}={ }^{\prime} \mathrm{O} \chi l$ 


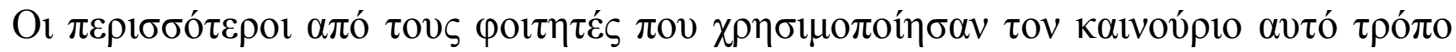

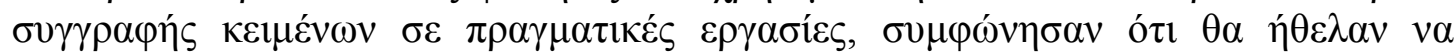

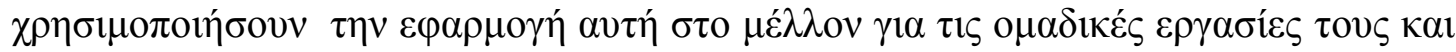

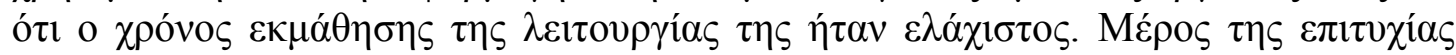

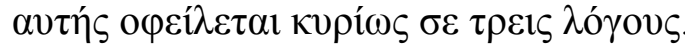

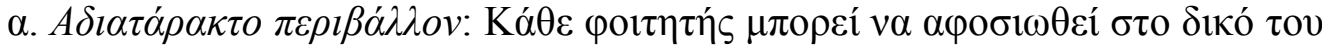

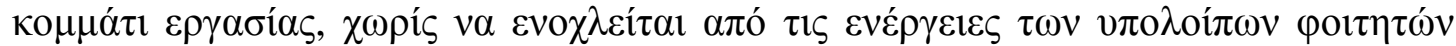

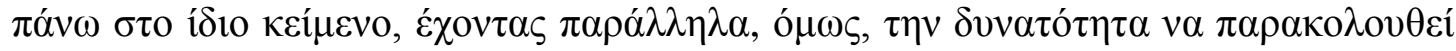
$\tau \eta \nu \varepsilon \xi \dot{\varepsilon} \lambda \imath \xi \eta \tau \eta \varsigma \varepsilon \rho \gamma \alpha \sigma i ́ \alpha \varsigma$.

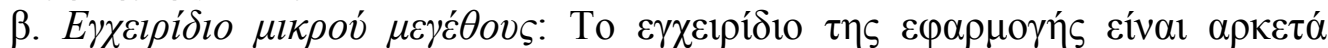

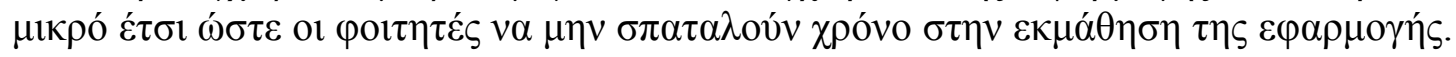

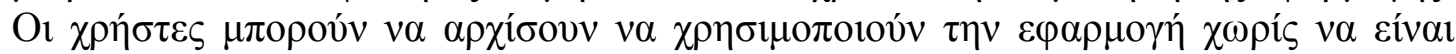

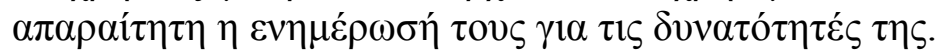

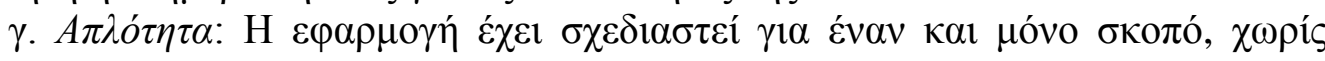

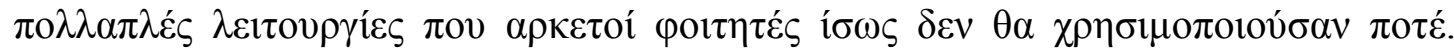

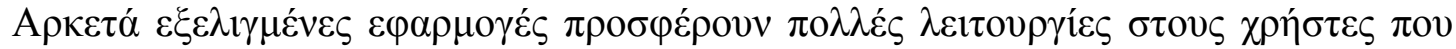

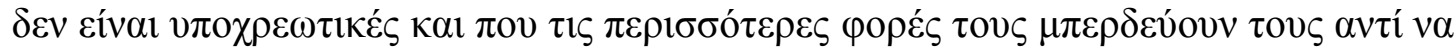

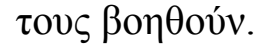

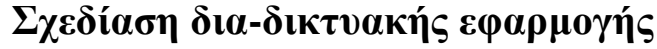

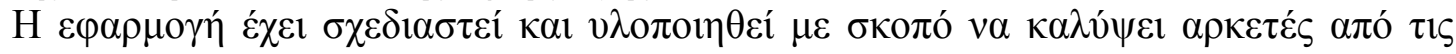

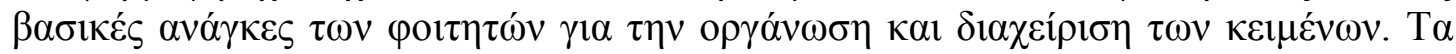

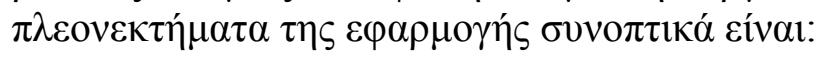

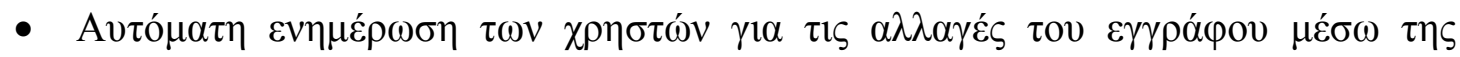

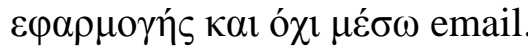

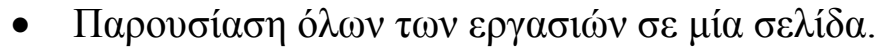

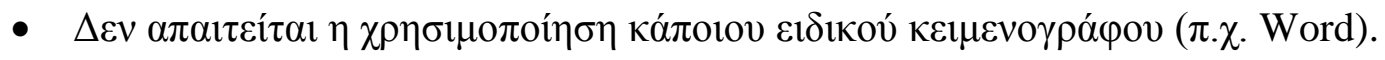

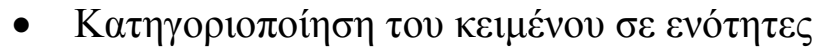

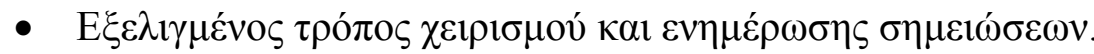

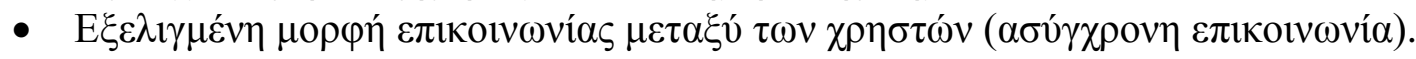

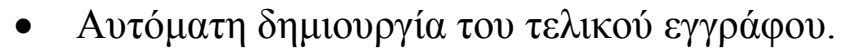

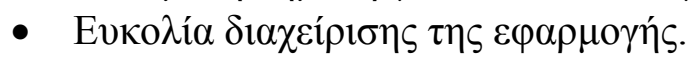

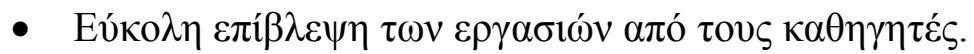

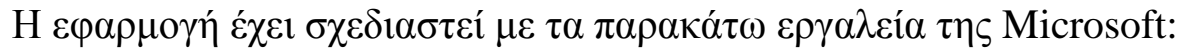

- Visual Studio 2010 express edition

- Sql Server 2008 express edition

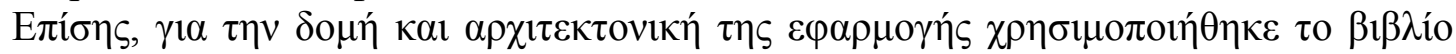
ASP .NET Web Developer's Guide.

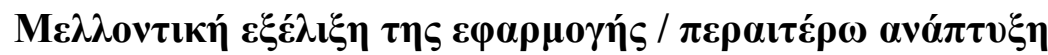

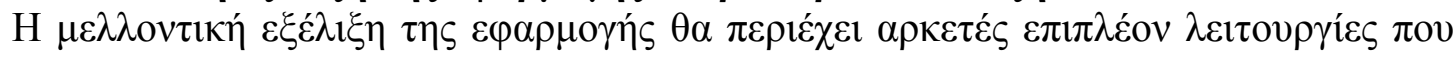

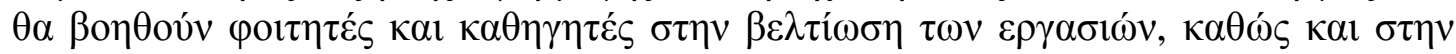

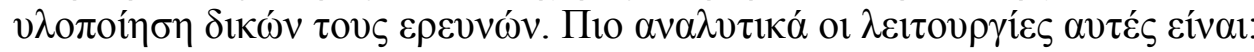

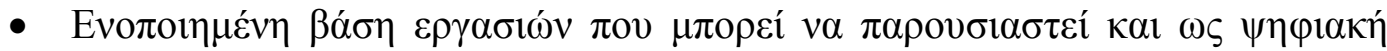

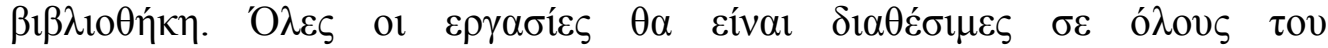

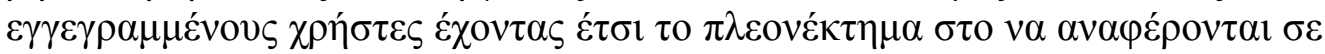

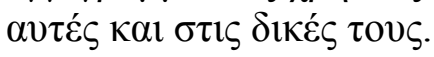




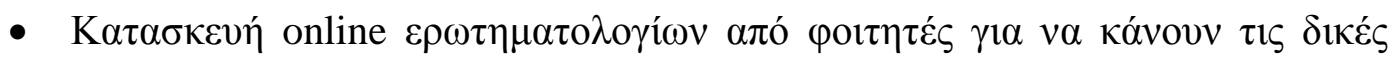

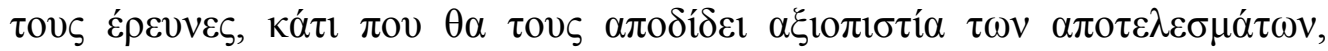

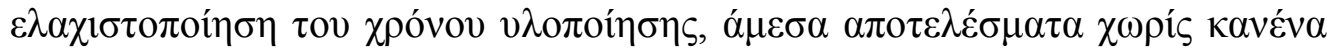

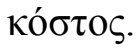

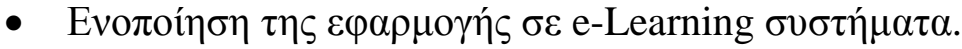

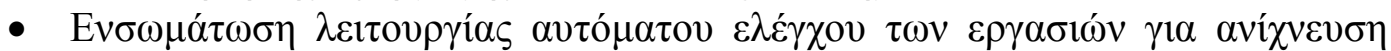

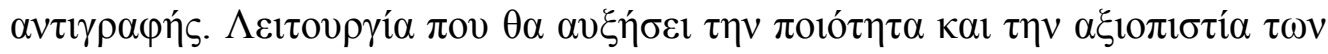

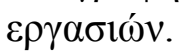

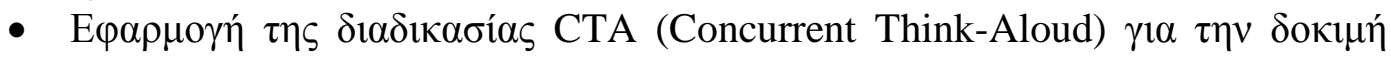

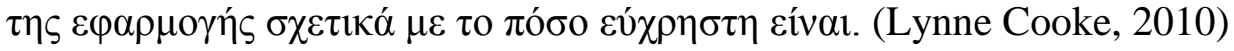

\section{$\Sigma v \mu \pi \varepsilon \rho \alpha \sigma \mu \alpha \tau u \kappa \alpha ́$}

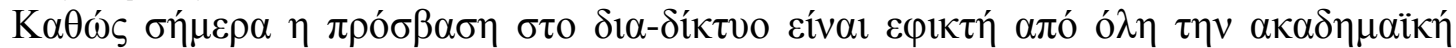

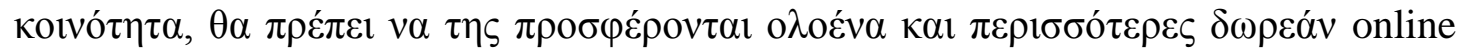

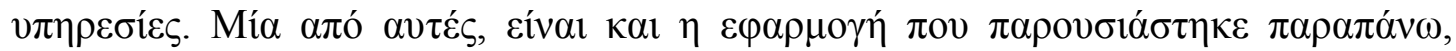

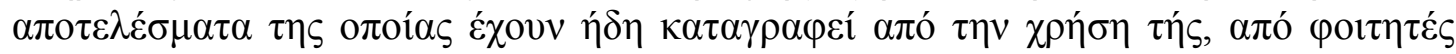

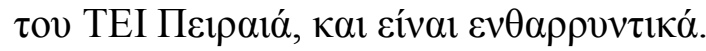

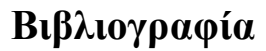

Noël, S. and Robert J.M., (2004). Empirical Study on Collaborative Writing: What Do Co-authors Do, Use, and Like?, The Journal of COMPUTER SUPPORTED COOPERATIVE WORK (CSCW), Retrieved on 25 July, 2010from http://www.springerlink.com/content/p0867n5746730504/

Hee-Cheol (Ezra) Kim and Kerstin Severinson Eklundh (2001). Reviewing Practices in Collaborative Writing, The Journal of COMPUTER SUPPORTED COOPERATIVE WORK (CSCW), Retrieved on 25 July, 2010 from http://www.springerlink.com/content/n337174702882827/

Cooke, L. (2010). Assessing Concurrent Think-Aloud Protocol as a Usability Test Method: A Technical Communication Approach, The Journal of IEEE Transactions on Professional Communication, Retrieved on 15 May, 2011 from http://ieeexplore.ieee.org/xpl/freeabs_all.jsp?isnumber=5556396\&arnumber $=5556472$

Hannes Olivier and Niels Pinkwart (2009). Towards Supporting Phases in Collaborative Writing Processes, in Yuhua Luo (eds.), Lecture Notes in Computer Science, Retrieved on 25 May, 2011 from http://ieeexplore.ieee.org/xpl/freeabs_all.jsp?isnumber=5556396\&arnumber=55564 72

Mesbah Ahmed, Chris Garrett, Jeremy Faircloth, Chris Payne (2002). ASP .NET Web Developer's, Editors: Wei Meng Lee, Jonothon Ortiz 